\title{
Seeding the Formation of Cold Gaseous Clouds in Milky Way-size Halos
}

\section{Citation}

Kereš, Dušan, and Lars Hernquist. 2009. "SEEDING THE FORMATION OF COLD GASEOUS CLOUDS IN MILKY WAY-SIZE HALOS." The Astrophysical Journal 700 (1): L1-5. https:// doi.org/10.1088/0004-637x/700/1/L1.

\section{Permanent link}

http://nrs.harvard.edu/urn-3:HUL.InstRepos:41381634

\section{Terms of Use}

This article was downloaded from Harvard University's DASH repository, and is made available under the terms and conditions applicable to Open Access Policy Articles, as set forth at http:// nrs.harvard.edu/urn-3:HUL.InstRepos:dash.current.terms-of-use\#OAP

\section{Share Your Story}

The Harvard community has made this article openly available.

Please share how this access benefits you. Submit a story.

Accessibility 
Draft VERSion OCTOBER 30, 2018

Preprint typeset using IATEX style emulateapj v. 04/20/08

\title{
SEEDING THE FORMATION OF COLD GASEOUS CLOUDS IN MILKY WAY SIZE HALOS
}

\author{
DUŠAN KEREŠ \& LARS HERNQUIST \\ Harvard-Smithsonian Center for Astrophysics, Cambridge, MA 02138; dkeres@cfa.harvard.edu \\ Draft version October 30, 2018
}

\begin{abstract}
We use one of the highest resolution cosmological SPH simulations to date to demonstrate that cold gaseous clouds form around Milky Way size galaxies. We further explore mechanisms responsible for their formation and show that a large fraction of clouds originate as a consequence of late-time filamentary "cold mode" accretion. Here, filaments that are still colder and denser than the surrounding halo gas, are not able to connect directly to galaxies, as they do at high redshift, but are instead susceptible to the combined action of cooling and Rayleigh-Taylor instabilities at intermediate radii within the halo leading to the production of cold, dense pressure-confined clouds, without an associated dark matter component. This process is aided through the compression of the incoming filaments by the hot halo gas and expanding shocks during the halo buildup. Our mechanism directly seeds clouds from gas with substantial local overdensity, unlike in previous models, and provides a channel for the origin of cloud complexes. These clouds can later "rain" onto galaxies, delivering fuel for star formation. Owing to the relatively large cross section of filaments and the net angular momentum carried by the gas, the clouds will be distributed in a modestly flattened region around a galaxy.
\end{abstract}

Subject headings: galaxies: formation - Galaxy: formation - Galaxy: halo — instabilities - methods: numerical

\section{INTRODUCTION}

Observations of neutral hydrogen around the Milky Way (MW) have revealed that our Galaxy is surrounded by a large number of cold gaseous clouds. Owing to their high velocities compared to galactic rotation, it was proposed that these objects reside outside the disk (Muller et al. 1963), and they became known as highvelocity clouds (HVCs). The masses of most HVCs are uncertain because of their uncertain distances (several HVC complexes are now placed at $D \sim 10 k p c$ (Wakker et al. 2008; Thom et al. 2008)), but are estimated to be $10^{5}-10^{7} M_{\odot}$ (Wakker \& van Woerden 1997). Similar HI clouds and cloud complexes have been found around other galaxies (for a review see Sancisi et al. 2008).

The overall kinematics of HVCs implies net inflow, motivating a picture where HVCs represent the building blocks of our Galaxy (Oort 1970) and provide the supply of gas needed for its relatively high star formation rate. In fact, our Galaxy has been forming stars at a steady rate for the last several Gyrs (Binney et al. 2000), implying that the gas consumed must be continuously replenished.

Many HVCs can be ionized by the external UV field, so HI observations likely detect only the densest of these structures that are able to self-shield. The presence of clouds around other galaxies is also implied by absorption line studies at both low and intermediate redshifts (Lanzetta \& Bowen 1990; Gauthier et al. 2009), which typically find gaseous clouds to distances of several tens of kiloparsecs in a halo. It is likely that these absorbing clouds are analogs to the local HVC population (Maller \& Bullock 2004).

Despite numerous theories, the origin of these clouds remains uncertain. Proposed mechanisms fall into the following three classes: 1: Cooling instabilities in the hot medium (Field 1965; Mo \& Miralda-Escude 1996) that can interact with the hot halo gas resulting in net infall onto the Milky Way (Maller \& Bullock 2004). 2: Production by a "galactic fountain" where outflows driven by supernovae from the disk condense in the hot corona and fall back onto the MW (Bregman 1980), possibly entraining hot gas from the halo (Fraternali \& Binney 2008). 3: Interaction of hot halo gas with infalling satellites (e.g. Putman et al. 2003), to account for clouds with specific properties; e.g. those in the Magellanic Cloud system. In principle, all of these channels could operate, complicating modeling and data interpretation. Here, we concentrate on cloud formation from cooling and hydrodynamical instabilities in infalling gas.

Cosmological hydrodynamic simulations have shown that at high redshift the bulk of the gas supplied to galaxies is accreted from the intergalactic medium through filaments in a "cold mode" (Katz et al. 2003; Kereš et al. 2005; Keres 2007; Kereš et al. 2009; Dekel \& Birnboim 2006; Ocvirk et al. 2008; Brooks et al. 2009) even after a hot halo atmosphere develops. Owing to lower densities, at late times there is a shift in the physics that governs the accretion, where filamentary flows get disrupted in the central parts of massive halos. We examine this late-time accretion and show that these disrupted filaments are still able to supply cold gas to MW size galaxies. We demonstrate that accretion through filaments creates density inversions in halos, triggering a combination of cooling and Rayleigh-Taylor (RT hereafter) instabilities that produce clouds, supplying MW like galaxies with gas. Earlier work hinted that the "leftovers" from filamentary might contribute to cloud formation (Sommer-Larsen 2006; Connors et al. 2006; Peek et al. 2008; Kereš et al. 2009) but did not demonstrate why and how this process operates.

\section{SIMULATIONS}


We use the Gadget-2 Smoothed Particle Hydrodynamics (SPH) code (Springel 2005) which simultaneously conserves energy and entropy, preventing numerical phase mixing. This is potentially an important property for modeling cloud formation as many SPH implementations can mix cold, dense and hot, dilute phases (Springel \& Hernquist 2002; Keres 2007). Gravitational forces are calculated combining Particle Mesh and hierarchical tree algorithms. In order to capture the formation of $\sim 10^{6} M_{\odot}$ clouds at high spatial resolution in a large volume, we adopt a zoom-in technique, where we first simulate a cosmological box of $10 h^{-1} \mathrm{Mpc}$ on a side using only dark matter (DM hereafter) particles. Next, we select a region that will collapse into a MW size object by $z=0$. We populate this region and its surroundings at high resolution with gas and DM particles, introducing $\Lambda \mathrm{CDM}$ density fluctuations down to the new inter-particle separation scale. Outside of this region, the density field is sampled more coarsely with high mass DM particles to provide long range forces. Such initial conditions are re-generated at $z=99$ using the Eisenstein \& Hu (1999) power spectrum in a $\Lambda \mathrm{CDM}$ cosmology: $\Omega_{b}=0.044, \Omega_{m}=0.27, h=0.7, \sigma_{8}=0.8$. We include standard cooling processes for a gas of primordial composition (Katz et al. 1996) and the UV background (Haardt \& Madau 1996). For simplicity, we neglect galactic outflows.

In total, the simulation has 6.6 million particles. The most massive halo in the high-resolution region (hereafter, $\mathrm{MWH}$ ), analyzed in detail in what follows, at $\mathrm{z}=0$ has $M_{\text {halo }}=7 \times 10^{11} M_{\odot}$ and contains 1.4 million baryonic and 0.9 million DM particles. Each gas particle begins with a mass of $9 \times 10^{4} h^{-1} M_{\odot}$, while stars are half this mass. Gravitational softening is the same for DM and baryonic particles, $\epsilon=400 h^{-1}(1+z)^{-1}$ pc (Plummer equivalent).

\section{RESULTS}

\subsection{Clouds}

In Figure 1, we show the inner regions of MWH. Gas particles with $n_{H}>4 \times 10^{-4} \mathrm{~cm}^{-3}$ are plotted close to face-on and edge-on projections of the disk, revealing the presence of a population of dense gaseous clouds together with a central galactic disk. Plotting gas colder than $30000 \mathrm{~K}$ selects an identical cloud population. At low redshift MWH contains a large number of cold, $T_{c} \sim 10^{4} \mathrm{~K}$, dense clouds in a $40 h^{-1} \mathrm{kpc}$ (edge on) times $60 h^{-1} \mathrm{kpc}$ (face on) region around the disk. We plot gas particles with small arrows to indicate the velocity field, demonstrating that the clouds exhibit a global sense of rotation, similar in orientation to the outer disk.

We identify clouds with $T<30000 \mathrm{~K}$ and $n_{H}>0.004$ $\mathrm{cm}^{-3}$ using the code SKID. The results are mainly determined by the temperature threshold and are robust for any $T<10^{5} \mathrm{~K}$. To distinguish clouds from galaxies we modified SKID to include thermal support of gas to properly identify self-gravitating systems. Bound objects include the main galactic disk, several satellite galaxies (one visible in the upper left corner of Figure 11), and a small number of gaseous clumps that are not associated with a DM or stellar component. These self-gravitating clouds are close to the halo center, where the external pressure compresses them to high densities. For sim- plicity, we exclude all bound objects from the remainder of the analysis, but apart from the main gaseous disk they contribute negligibly to the cold gas budget. Cloud masses range from $10^{6}$ to few $10^{7} M_{\odot}$. We note, however, that we are limited at low masses by the simulation resolution. Some of the most massive clouds are at larger radii and often represent a dense slab of gas, which might be susceptible to further instabilities. Typical column densities (for the total cold gas, i.e. no ionization correction which could be substantial) of clouds are $N_{H} \sim 5 \times 10^{18}-10^{21} \mathrm{~cm}^{-2}$.

\subsection{Gas in the inner halo}

The amount of virialized gas increases with time as the halo grows and hot gas fills low density regions inbetween colder, denser filaments (Kereš et al. 2005). At high redshift, filaments survive within hot halos even when the hot medium dominates the halo gas budget $\left(M_{h}>3 \times 10^{11} M_{\odot}\right)$. However, at lower redshift the density of filaments decreases and the temperature increases so they are more easily destroyed at small radii by large scale shocks in a hot halo. This happens where halo shock conditions are favorable for hot, overdense gas to propagate outwards (Birnboim \& Dekel 2003). Filamentary remnants that penetrate into halos but not quite to galaxies set the stage for cloud formation. For the MWH this epoch starts roughly at $z=1-1.5$ after the last major merger is completed. The last significant $(\sim 1: 7)$ merger occurs around $\mathrm{z}=0.5$ and soon afterwards $\mathrm{MWH}$ reaches a quasi-static configuration that lasts 2-3 Gyrs, although cloud formation proceeds in a similar manner for $z<1$. At $z \sim 0$ around 10 percent of the halo gas (excluding gas in the galaxies) within the inner $100 h^{-1} \mathrm{kpc}$, $1.5-2 \times 10^{9} M_{\odot}$, is in cold gaseous clouds as a result of a balance between cloud creation, accretion and destruction. This is consistent with estimates of the total mass of the HVC population (Putman 2006). The density of the hot component at a distance of $50 \mathrm{kpc}$ from the main galaxy is $n_{H} \sim 0.5-1.5 \times 10^{-4} \mathrm{~cm}^{-3}$, consistent with observational limits (Grcevich \& Putman 2009).

\subsection{The origin of the cold clouds}

To understand their origin we follow cloud particles backwards in time and determine the maximum temperature that they had in the past, $T_{\max }$. This tells us if the clouds form through instabilities in a hot corona or if they were seeded by colder structures. Figure 2 shows the results of this analysis (for "resolved" clouds, $\geq 32$ particles). Most of the mass in the clouds never reached $0.5-1.5 \times 10^{6} \mathrm{~K}$, characteristic of the MWH at $\mathrm{z}=0$, but instead clouds form from, $1-1.5 \times 10^{5} \mathrm{~K}$ material, before they cool to $\sim 10^{4} \mathrm{~K}$. These are temperatures characteristic of low-redshift, large-scale filaments fueling gas onto MWH. The distribution of $T_{\max }$ is similar in the redshift interval $0<z<1$, implying a connection between cloud formation and "cold mode" accretion active at higher redshifts.

We illustrate this process in Figure 3. An overdense, warm filament penetrates to intermediate radii in the halo, visible in the panels which show density and temperature. The filament is not directly connected to the galaxy but manages to reach $30-40 \mathrm{kpc}$ radius before being heated to higher temperatures. The large impact 


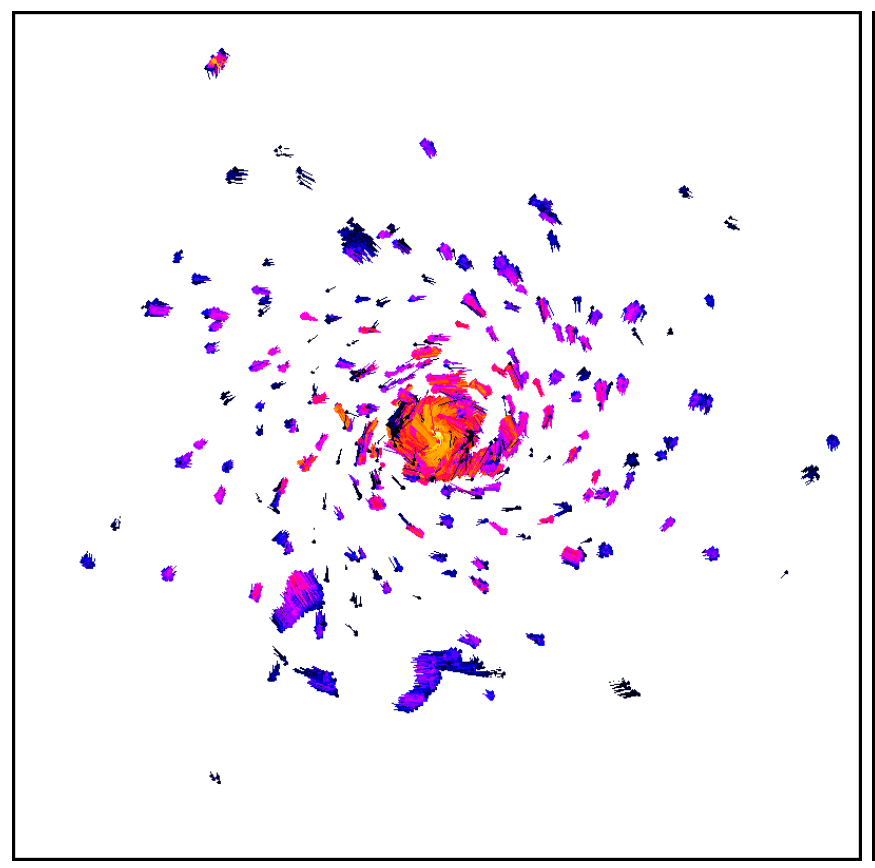

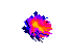

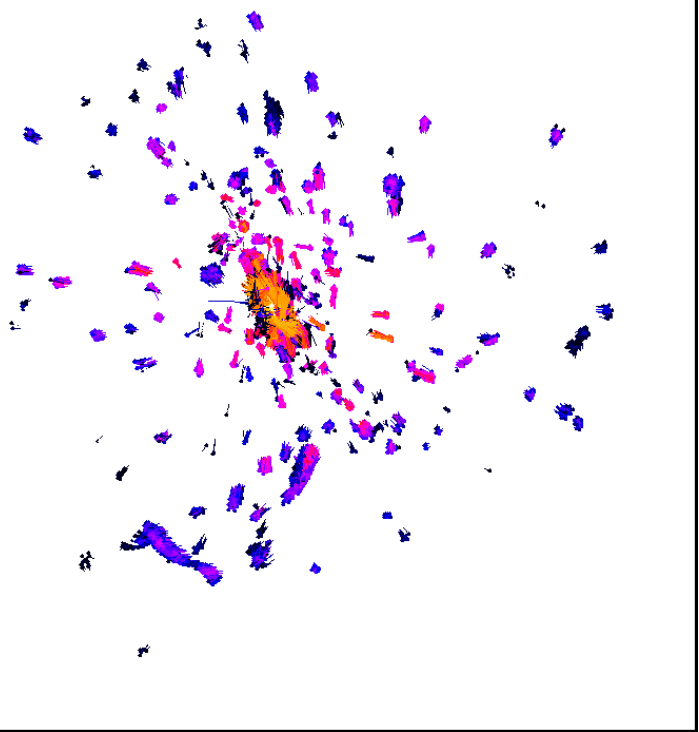

FIG. 1.- Distribution of dense clouds in a $200 h^{-1} \mathrm{kpc}$ box centered on the most massive galaxy in MWH. Particles are color coded by density from $n_{H}=4 \times 10^{-4} \mathrm{~cm}^{-3}$ (darkest blue) to $4 \mathrm{~cm}^{-3}$ (bright yellow). Clouds have temperatures $T_{c} \sim 10^{4} \mathrm{~K}$.

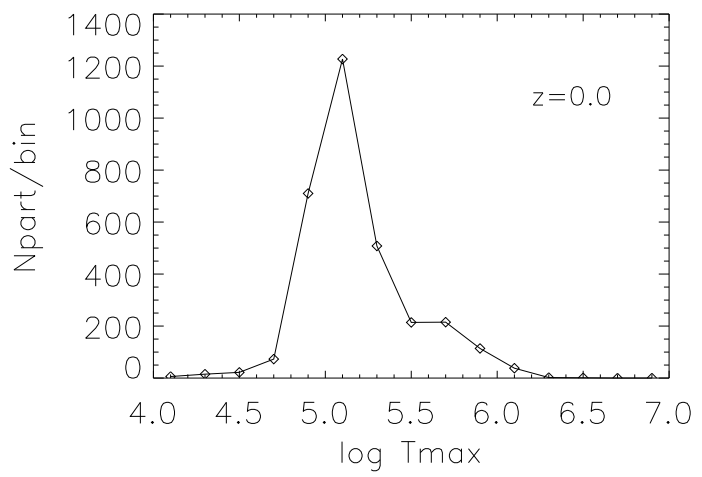

FIG. 2.- Histogram of the maximum temperature cloud particles reached before condensing into clouds, in bins of $0.2 \log T_{\max }$.

parameter with respect to the central galaxy creates a density inversion where "heavier" fluid lies on top of a "lighter" one in an external gravitational field (of the parent halo), providing the conditions for RT instabilities to operate. The initial instabilities that act on overdense filamentary gas often produce cometary taillike morphologies, similar to RT instabilities in the ISM (e.g. O'dell \& Burkert 1997). These are further compressed by higher pressure gas at slightly smaller radii and quickly become cooling unstable, forming cold, dense clouds. Additional sites of cloud formation throughout the MWH can be seen in the figure, often where compression of infalling gas by halo shocks and pressure from surrounding hot gas promotes faster development of instabilities.

The filament is also moving with respect to the surrounding lower density gas. It is therefore possible that Kelvin Helmholtz $(\mathrm{KH})$ instabilities can affect this configuration. It has been shown that $\mathrm{SPH}$ techniques have difficulty handling KH instabilities (Agertz et al. 2007) so it is important to estimate the timescales for a dominant mode to affect and destroy such penetrating filaments before they become cooling or RT unstable at small radii. The relevant timescale is:

$$
t_{K H}=\frac{\lambda\left(\rho_{c}+\rho_{h}\right)}{\left(\rho_{c} \rho_{h}\right)^{1 / 2} U} \sim \lambda\left(\rho_{c} / \rho_{h}\right)^{1 / 2} / U,
$$

where $\rho_{c}$ and $\rho_{h}$ are the densities of the heavier and more dilute components, respectively, $\lambda$ is the spatial scale of the instability, and $U$ is the relative velocity of the two phases. For a spherical cloud moving in a dilute medium the disruption time is $2-5 \times t_{K H}$ (self-gravity can make it even longer) (Murray et al. 1993). At the temperatures typical of penetrating filaments in the $z \sim 0.5 \mathrm{MWH}$, $T \sim 10^{5} \mathrm{~K}$ which for approximate pressure equilibrium implies a density contrast of $\sim 5-10$. The overdense region in Figure 3 moves with velocities $\sim 100 \mathrm{~km} / \mathrm{s}$ through the hot medium. The largest available mode is responsible for the destruction of structures. The length of the structure is $\sim 100 \mathrm{kpc}$ and its typical width is $\sim 20$ kpc. Assuming a $3 \times t_{K H}$ case for a wavelength comparable to the filament's width, it takes about $2 \times 10^{9}$ yrs for the instability to act. This is longer than the time over which the filament is destroyed by shocks and other instabilities and the time needed for gas to flow through the instability region visible in Figure 3. It is also possible that the filament length is relevant which increases this timescale by factor of few. We therefore conclude that this structure can indeed penetrate into a hot halo to create such a configuration. Only much smaller modes can affect the infalling cold gas in a filament, perhaps enhancing the surface shearing layer.

Next, we estimate the timescale for RT instabilities (Murray et al. 1993) to act:

$$
t_{R T}=2 \pi\left[\frac{2 \pi g}{\lambda}\left(\frac{\rho_{c}-\rho_{h}}{\rho_{h}+\rho_{c}}\right)\right]^{-1 / 2},
$$



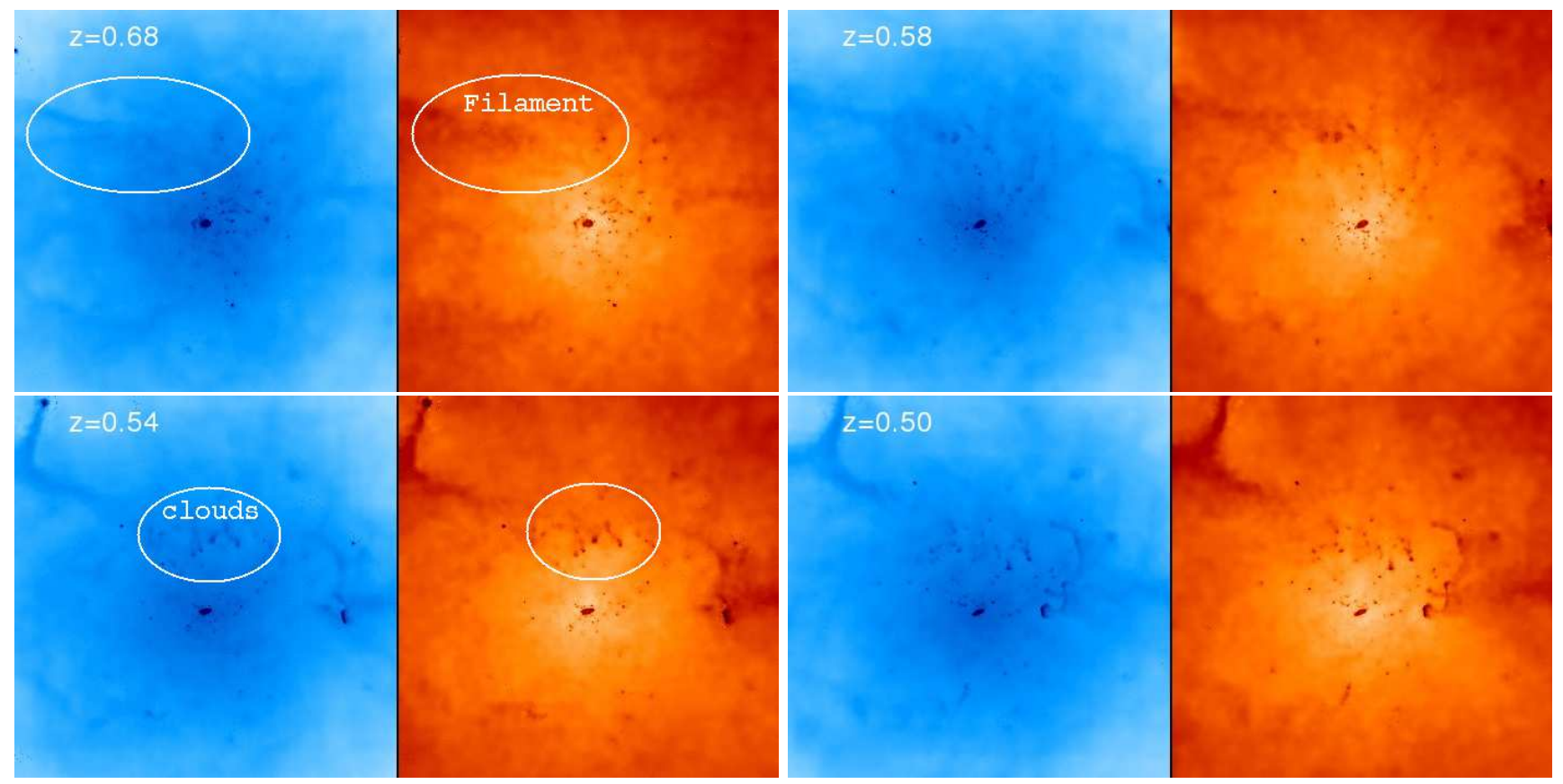

FIG. 3. - Example of the instability developing from an overdense filament in a hot dilute halo gas leading to cloud formation. Left panels show the projected density and the right panels show projected mass-weighted temperature in a box $200 h^{-1} \mathrm{kpc}$ on a side and $100 h^{-1} \mathrm{kpc}$ thick.

where $g$ is the gravitational acceleration. Taking the acceleration at $50 \mathrm{kpc}$ in $\mathrm{MWH}$ and a density contrast $\sim 5$, we estimate the timescale for the instability at $\sim 5 \mathrm{kpc}$ scales, which corresponds to the example in Figure 3 . $t_{R T} \sim 3.5 \times 10^{8} \mathrm{yr}$, comparable to $t_{\text {cool }} \sim 3-4 \times 10^{8}$ yr of the photo-ionized gas in a warm filament at $T \sim$ $1-1.2 \times 10^{5} \mathrm{~K}$ and $n_{H}=2 \times 10^{-4} \mathrm{~cm}^{-3}$. Therefore, RT and cooling instabilities are acting together to form pressure supported clouds with $T_{c} \sim 10^{4} \mathrm{~K}$. Both of these timescales are shorter than the time over which the overdense structure is present in the inner halo. This process often simultaneously forms many clouds in the same region, yielding cloud complexes. At this stage, $\mathrm{KH}$ instabilities of similar wavelength could play a role. When clouds start to form, shearing velocities are slowed down by pressure gradients and shocks before they accelerate to terminal velocity so the role played by $\mathrm{KH}$ instabilities is unclear.

\section{DISCUSSION}

Halos formed in fully cosmological environments are complex and dynamic systems. In addition to the processes described above, infalling satellites can be stripped of gas as they pass through overdense filaments in the outskirts of halos, providing material for further instabilities and cloud formation. This effect can alleviate the need for a high density hot medium to produce large gaseous tails such as the one observed in the Magellanic Cloud system (Putman et al. 2003). An example of this is visible in the upper left corners of the bottom two panels in Figure 3. Infalling substructures create density waves and shocks in the halo, providing a source of further compression and acceleration which enables RT instabilities to develop even without the gravitational pull of the parent halo. Similarly, we expect density inversions and RT instabilities to be more common in sim- ulations with galactic outflows (both stellar and AGN), where winds can temporarily push, compress and accelerate both galactic and infalling material (Di Matteo et al. 2005).

The MWH has a growth history typical for its mass indicating that this cloud formation process is general. In future work we will discuss cloud formation in larger halos where clouds form at earlier times (e.g., see Figure 6 in Kereš et al. 2009). Halo clouds are likely part of halo absorption systems observed at intermediate redshift and some are likely analogs of the HVCs. However, from Figure 1 it is already clear that many of the cold clouds have velocities comparable to the galactic rotation which will make searches for such objects difficult (Peek et al. 2008). Cloud infall at large radii will also likely trigger star formation and provide kinematic enhancements in the outer disks.

Cloud formation from cooling instabilities in an idealized setting was simulated in Kaufmann et al. (2009), demonstrating that large entropy cores are needed for this mechanism to operate. This is supported by Binney et al. (2009) who examined relevant timescales for the onset of cooling instabilities that begin with small density enhancements and concluded that unless the entropy profile of the halo gas is shallow, buoyancy and conduction will prevent cloud formation. In our simulation clouds are seeded at intermediate radii starting from already moderate overdensities, for which this linear analysis does not apply. However, the inner gas entropy and density profiles of $\mathrm{MWH}$ are quite shallow, so it is not clear if slower cloud formation is prevented. Interestingly, clouds just below our resolution limit, $<32$ particles, do have a larger contribution from a hot gas component. More work is needed to determine if this is a physical effect or purely numerical if unresolved objects partially mix in hotter gas. In reality, even a moderate 
metallicity of halo gas could increase cloud formation efficiency.

Both SPH and Eulerian codes have shortcomings in following the instabilities relevant when clouds move in a hot medium. New techniques, such as the moving mesh code of Springel (2009), may avoid these shortcomings, providing new insights into the formation and evolution of HVCs.

\subsection{Survivability of clouds}

It is interesting to determine if clouds formed in $\mathrm{MWH}$ can survive their trip through the inner halo and rain onto the galaxy, providing fresh fuel for star formation. In the absence of magnetic fields, thermal conduction will directly evaporate clouds smaller than about $M_{c} \sim 10^{5} M_{\odot}$ in a free fall time for the typical radii where clouds form in MWH (Mo \& Miralda-Escude 1996). The evolution of more massive clouds is likely governed by a combination of RT and KH instabilities (e.g. see Murray et al. 1993; Murray \& Lin 2004). Our simulations do not have sufficient resolution to follow instabilities on such small scales and SPH itself might not be suitable for this task.

We can, however, estimate relevant timescales. Idealized simulations show that clouds fragment when they sweep up a mass comparable to their own (Murray \& Lin 2004). Therefore, we integrate the descent of clouds on radial orbits, assuming they form at $70 \mathrm{kpc}$ and need to reach $5 \mathrm{kpc}$ to fall onto a galaxy. We use $T_{h}=10^{6} \mathrm{~K}$, $T_{c}=10^{4} \mathrm{~K}$ and a density profile $\rho \propto r^{-\beta}$. We take $\beta=0.65-0.9$ (bracketing the inner density profile of the $\mathrm{MWH}$; for simplicity we assume $T_{h}=$ const.), and normalize the density at $70 \mathrm{kpc}$.

We find that clouds above $2-3 \times 10^{5} M_{\odot}$ survive if clouds are compressed during their descent. This mass is $\sim 5$ times higher if the cloud radius remains fixed dur- ing infall. In both cases this is smaller than masses of the clouds formed in our simulation. We also directly integrate cloud orbits accounting for gravity and ram pressure, assuming that clouds have angular momentum similar to the disk gas. This only mildly increases the mass limit for cloud survival compared to radial infall. Therefore, massive clouds seeded in the inner halo are likely able to bring cold material to a central disk. In the non-wind simulations at $\mathrm{z}=0$ this yields $0.6 M_{\odot} / \mathrm{yr}$ of fresh gas accretion, but if galactic outflows are included this increases to $\sim 1 M_{\odot} /$ yr (because more material is saved for later times). For the MW, whose mass is factor of $\sim 2$ larger than the simulated $\mathrm{MWH}$, the rates are likely higher, providing enough fuel for continuous star formation over a several Gyr period.

\section{CONCLUSIONS}

Using a state-of-the-art numerical simulation of structure formation in a $\Lambda \mathrm{CDM}$ universe we follow the formation of small $\sim 10^{6} M_{\odot}$ cold, pressure confined clouds in a halo similar to the MW. Most clouds are not randomly formed from small fluctuations in a hot, $T \sim 10^{6}$, medium as has often been supposed. Instead, they originate from overdense, $T \sim 10^{5}$, filamentary gas within a hot halo at low redshift. Such gas creates a density and entropy inversion in a gravitational field, driving simultaneous cooling and RT instabilities. During their initial evolution, many clouds form from cometary structures while later evolution cannot be followed properly with our computational scheme. However, simple estimates indicate that a large fraction of such clouds could indeed survive their trip to the halo center and provide a supply of gas for late time star formation in galaxies.

We are grateful to V. Springel for allowing us to use his code and to G. Besla, Y. Birnboim, A. Burkert and T. Cox for useful comments.

\section{REFERENCES}

Agertz, O., et al. 2007, MNRAS, 380, 963, arXiv:astro-ph/0610051

Binney, J., Dehnen, W., \& Bertelli, G. 2000, MNRAS, 318, 658, arXiv:astro-ph/0003479

Binney, J., Nipoti, C., \& Fraternali, F. 2009, ArXiv e-prints, 0902.4525

Birnboim, Y., \& Dekel, A. 2003, MNRAS, 345, 349, astro-ph/0302161

Bregman, J. N. 1980, ApJ, 236, 577

Brooks, A. M., Governato, F., Quinn, T., Brook, C. B., \& Wadsley, J. 2009, ApJ, 694, 396, 0812.0007

Connors, T. W., Kawata, D., Bailin, J., Tumlinson, J., \& Gibson, B. K. 2006, ApJL, 646, L53, arXiv:astro-ph/0509314

Dekel, A., \& Birnboim, Y. 2006, MNRAS, 368, 2, astro-ph/0412300

Di Matteo, T., Springel, V., \& Hernquist, L. 2005, Nature, 433, 604, astro-ph/0502199

Eisenstein, D. J., \& Hu, W. 1999, ApJ, 511, 5,

arXiv:astro-ph/9710252

Field, G. B. 1965, ApJ, 142, 531

Fraternali, F., \& Binney, J. 2008, ArXiv e-prints, 802, 0802.0496

Gauthier, J.-R., Chen, H.-W., \& Tinker, J. L. 2009, ArXiv e-prints, 0902.3237

Grcevich, J., \& Putman, M. E. 2009, ApJ, 696, 385, 0901.4975

Haardt, F., \& Madau, P. 1996, ApJ, 461, 20, astro-ph/9509093

Katz, N., Keres, D., Dave, R., \& Weinberg, D. H. 2003, in ASSL Vol. 281: The IGM/Galaxy Connection. The Distribution of Baryons at $\mathrm{z}=0$, ed. J. L. Rosenberg \& M. E. Putman, 185-+, astro-ph/0209279
Katz, N., Weinberg, D. H., \& Hernquist, L. 1996, ApJS, 105, 19, astro-ph/9509107

Kaufmann, T., Bullock, J. S., Maller, A. H., Fang, T., \&

Wadsley, J. 2009, MNRAS, 396, 191, 0812.2025

Keres, D. 2007, PhD thesis, University of Massachusetts Amherst

Kereš, D., Katz, N., Fardal, M., Davé, R., \& Weinberg, D. H. 2009, MNRAS, 395, 160, 0809.1430

Kereš, D., Katz, N., Weinberg, D. H., \& Davé, R. 2005, MNRAS, 363, 2, astro-ph/0407095

Lanzetta, K. M., \& Bowen, D. 1990, ApJ, 357, 321

Maller, A. H., \& Bullock, J. S. 2004, MNRAS, 355, 694, astro-ph/0406632

Mo, H. J., \& Miralda-Escude, J. 1996, ApJ, 469, 589, arXiv:astro-ph/9603027

Muller, C. A., Oort, J. H., \& Raimond, E. 1963, Academie des Sciences Paris Comptes Rendus, 257, 1661

Murray, S. D., \& Lin, D. N. C. 2004, ApJ, 615, 586, arXiv:astro-ph/0407411

Murray, S. D., White, S. D. M., Blondin, J. M., \& Lin, D. N. C. 1993, ApJ, 407, 588

Ocvirk, P., Pichon, C., \& Teyssier, R. 2008, MNRAS, 390, 1326, 0803.4506

O'dell, C. R., \& Burkert, A. 1997, in IAU Symposium, Vol. 180, Planetary Nebulae, ed. H. J. Habing \& H. J. G. L. M. Lamers, $332-+$

Oort, J. H. 1970, A\&A, 7, 381

Peek, J. E. G., Putman, M. E., \& Sommer-Larsen, J. 2008, ApJ, 674, 227, 0705.0357

Putman, M. E. 2006, ApJ, 645, 1164, arXiv:astro-ph/0603650 
Putman, M. E., Staveley-Smith, L., Freeman, K. C., Gibson, B. K., \& Barnes, D. G. 2003, ApJ, 586, 170, arXiv:astro-ph/0209127

Sancisi, R., Fraternali, F., Oosterloo, T., \& van der Hulst, T. 2008, A\&A Rev., 15, 189, 0803.0109

Sommer-Larsen, J. 2006, ApJL, 644, L1, arXiv:astro-ph/0602595

Springel, V. 2005, MNRAS, 364, 1105, astro-ph/0505010

. 2009, ArXiv e-prints, 0901.4107
Springel, V., \& Hernquist, L. 2002, MNRAS, 333, 649, astro-ph/0111016

Thom, C., et al. 2008, ApJ, 684, 364, 0712.0612

Wakker, B. P., \& van Woerden, H. 1997, ARA\&A, 35, 217

Wakker, B. P., et al. 2008, ApJ, 672, 298, 0709.1926 